\title{
L'Hospitalet de Llobregat, modelo de generalización del aprendizaje-servicio
}

\section{Roser Batlle}

Grupo Permanente ApS de I'Hospitalet de Llobregat

Red Española de Aprendizaje-Servicio

\section{Resumen}

Actualmente l'Hospitalet de Llobregat (España) lidera el movimiento de municipios españoles que han apostado por desarrollar una política pública de impulso al aprendizaje-servicio. Se trata de un liderazgo reconocido en Cataluña, en España y también en el resto del mundo. Pocas ciudades pueden exhibir más de 70 proyectos de aprendizaje servicio, sostenidos por una red ciudadana espesa y entusiasta, donde participan centros educativos, entidades sociales y profesionales de la administración pública. Ha mejorado sensiblemente la educación de los chicos y chicas así como la cohesión social en el municipio; y, por otro lado, cada actor educativo y social ha sacado provecho del hecho de implicarse. Conocer las claves de esta historia de éxito puede ser inspirador para otros municipios y otros países.

\section{Palabras clave}

Aprendizaje-servicio; políticas públicas; éxito educativo; cohesión social; ciudadanía. 


\title{
L'Hospitalet de Llobregat, model of generalization of service- learning
}

\begin{abstract}
Currently, the Catalonian municipality of I'Hospitalet de Llobregat is leading a group of Spanish municipalities that have opted to develop a public policy aimed at boosting service-learning. Their strong leadership is recognised not only in Catalonia and Spain, but also in the rest of the world. Few cities can boast more than 70 servicelearning projects, which are supported by a strong and enthusiastic citizen network and involve participation from educational centres, social entities and public administration officials alike. Educational quality and social cohesion have significantly increased in the municipality and, likewise, each educational and social agent has benefitted from being involved. Understanding the reasons behind this success story could prove inspiring for other municipalities and other countries
\end{abstract}

\section{Keywords}

Service-learning; public policy; educational success; social cohesion; citizenship. 


\section{Radiografía actual del aprendizaje-servicio en L'Hospitalet de Llobregat}

La experiencia de L'Hospitalet de Llobregat cuanto al desarrollo del aprendizaje-servicio llama poderosamente la atención, es reclamada en diversos foros y la ciudad es visitada por pedagogos de otros países.

A día de hoy se han inventariado 106 proyectos estables de ApS. Estos proyectos han sido protagonizados por más de 2.000 alumnos de 44 centros educativos de primaria y secundaria en atención a las necesidades de los colectivos que acogen 70 entidades sociales y educativas de la ciudad (ONG, centros de personas discapacitadas, educación en el tiempo libre, residencias de personas mayores...).

Existe ya una aproximación registrada por el propio ayuntamiento (Graells, Esteve y Batlle, 2017) en relación al impacto educativo, que se concreta en:

a) El descenso del absentismo. Un elemento que ha sido evidente para el profesorado, especialmente los días en que los alumnos hacen su trabajo comunitario, experimentando a la vez una mejora del nivel de convivencia en las aulas

b) El descenso de la conflictividad. Este queda reflejado en los datos del servicio municipal de mediación, pasando de 52 intervenciones por conflicto entre escolares el curso 2009/10 a tan solo 5 intervenciones en el curso 2014/15. En estos datos tiene mucho que ver uno de los programas estrella del ApS en la ciudad, que forma cada curso a un centenar de alumnos mediadores en sus propios centros educativos.

c) El nivel de graduación en Educación Secundaria. Un nivel que ha experimentado un aumento de 9 puntos, pasando del $70 \%$ al $79 \%$. Asimismo, el nivel de alumnos con excelencia en las pruebas de acceso a la Universidad se ha multiplicado por 8.

Aunque no sería exacto atribuir exclusivamente al aprendizaje-servicio todos estos buenos datos, los responsables municipales están convencidos de la influencia en ellos del aprendizaje-servicio, un elemento que mejora la calidad de la tarea docente al involucrar a otros agentes educativos.

\section{2. ¿Por qué I'Hospitalet ha sido terreno abonado para el ApS?}

En Cataluña, desde el año 2003, el aprendizaje-servicio se ha extendido como la pólvora, en gran parte porque muchos centros educativos y muchas entidades sociales ya lo practicaban sin bautizarlo, conceptualizarlo o sistematizarlo.

La creación en 2004 del Centro Promotor de Aprendizaje Servicio, una red de personas y entidades impulsada por la sociedad civil, dio un fuerte impulso a su difusión en todos los niveles educativos y en todo tipo de instituciones educativas. Pronto los municipios se interesaron por el ApS, porque lo veían como una buena estrategia en el marco de las políticas 
de promoción del éxito educativo y la cohesión social. En l'Hospitalet de Llobregat el contagio del ApS fue fulminante.

L'Hospitalet de Llobregat es la segunda ciudad más poblada de Catalunya con más de 260.000 habitantes en solo $12,4 \mathrm{Km} 2$ y se encuentra pegada a Barcelona. Si en el siglo XIX todavía era una pequeña población agrícola y en el siglo XX pasó a ser una ciudad industrial, actualmente es sobre todo una ciudad eminentemente de servicios.

La ciudad está compuesta por barrios de fuerte personalidad, tanto urbanística como social y cultural, aunque podríamos decir que, en líneas generales, se trata de una ciudad con amplia proporción de clase obrera. El $24 \%$ de su población es menor de 25 años y el $21 \%$ es mayor de 65 años. Está dividida por el paso de las vías férreas que separan el norte y el sur de la ciudad. Los barrios del norte son los más densamente poblados, llegando a 70.000 habitantes por $\mathrm{km} 2$. Son también los barrios que han recibido la mayor parte de la población migrada y los más castigados por la crisis y el paro.

Los casi 50.000 vecinos que nacieron en el extranjero Ilegaron a I'Hospitalet en la última década, procedentes, sobre todo, desde Bolivia, Marruecos, Ecuador, Paquistán y República Dominicana. En conjunto, la población inmigrante es del $19 \%$ pero su distribución en la ciudad es desigual y en algunos barrios supera el 35\%. En mayo del año 2018 la tasa de desempleo de l'Hospitalet fue del
10,7\%. En Cataluña, en este mismo período, el desempleo más elevado fue un $13,29 \%$ y el más bajo un $5,24 \%$.

Los habitantes de I'Hospitalet han sufrido muy duramente los efectos de la crisis económica. Por poner un ejemplo, en el año 2012, se alcanzó un $19 \%$ de desempleo. Al igual que en muchas otras ciudades españolas y catalanas, en ese mismo periodo hubo importantes recortes económicos en salud, educación, servicios sociales y dependencia, por parte de los gobiernos estatal y autonómico. Sin embargo, el ayuntamiento de I'Hospitalet priorizó las partidas sociales en los presupuestos municipales y esta voluntad política contribuyó a compensar, al menos en parte, el impacto de la crisis.

\section{Las raíces del aprendizaje- servicio en la ciudad}

En este apartado se presentarán cuáles han sido las actividades existentes en la ciudad que, a modo de precedentes, han favorecido la posterior aparición del aprendizaje-servicio para centrarnos a continuación en aquellos proyectos nuevos o ya existentes que se perciben claramente como actividades de aprendizaje-servicio.

Los precedentes: una trama de proyectos solidarios

La historia pedagógica de la ciudad cuenta con muchas experiencias de escuelas y esplais (centros de educación en el tiempo libre) que tradicionalmente han impulsado proyectos solidarios con los chicos y 
chicas, al considerarlos básicos para su formación humana. Sin embargo, antes de 2004, prácticamente nadie bautizaba estos proyectos como prácticas de aprendizaje-servicio. De hecho, muchos de ellos eran concebidos y presentados como proyectos de voluntariado, dentro de la escuela o dentro de los esplais.

Pero tarde o temprano muchas acciones de voluntariado suelen ser inspiradoras de experiencias de aprendizaje-servicio para las escuelas. Cuando éstas se plantean reforzar los vínculos con los contenidos educativos o curriculares, las acciones solidarias se transforman poco a poco en proyectos de aprendizaje-servicio.

Una de las experiencias veteranas y precedentes es la de los Encuentros por la Tolerancia del Centro Educativo Fax (barrio de Can Serra) que comenzaron en 1996, en el que suelen participar unos 30 alumnos de educación secundaria obligatoria de este centro y unas 70 personas adultas del Centro Ocupacional Can Serra. Se trata de un proyecto bidireccional, donde todo el mundo aprende y todo el mundo da un servicio a otro, a partir del trabajo en torno a un centro de interés común.

Otra experiencia histórica referente, antes de 2004, fue el crédito de Solidaridad y Valores del Centro de Estudios Joan XXIII (barrio de Bellvitge), ubicado inicialmente dentro de la materia de Ética, y posteriormente dentro de Ética y Ciudadanía de $4^{\circ}$ de Educación Secundaria Obligatoria. A partir de los contenidos curriculares de la asignatura, los chicos y chicas eligen entre hacer un trabajo teórico sobre alguna organización no gubernamental o entidad de voluntariado o bien hacer un servicio de voluntariado de 2 horas semanales en alguna entidad social del entorno. Entre un $70 \%$ y un $80 \%$ de los jóvenes eligen esta opción.

De hecho, este centro educativo llevaba un largo camino de acciones solidarias. Una de las de mayor impacto fue en el verano de 1997, la expedición solidaria en Tuzla, una iniciativa de los

estudiantes de último año de Formación Profesional de la rama de Electricidad, para montar calentadores de agua a casas de los afectados por la guerra de Bosnia. Si bien estaba planteada como acción de voluntariado, representaba al mismo tiempo una práctica profesional solidaria para los futuros electricistas.

Por otra parte, también hay que contar como precedente el Consejo Municipal de Chicos y Chicas de la ciudad que, en el formato actual, se inició en 2003. Si bien las iniciativas de consejos municipales de niños no nacen en Cataluña inspiradas en el aprendizajeservicio, en la práctica responden a este concepto, dado que los chicos y chicas se forman actuando como ciudadanos comprometidos en diferentes asuntos de su entorno.

Finalmente, los esplais de la ciudad (Club Infantil Juvenil Bellvitge, Club d'Esplai Pubilla Casas-Can Vidalet, Club Sanfeliu, Esplai La Florida, Club de Esplai Can Serra, Asociación Educativa Itaca ...) han llevado a cabo, como mínimo desde 1974, actividades de aprendizaje-servicio con el formato de 
campos de trabajo en verano. Han sido actuaciones de conservación del medio ambiente, de restauración del patrimonio o de difusión cultural, en general a lugares alejados de l'Hospitalet de Llobregat.

Enmarcados en la pedagogía del proyecto, propia de los movimientos scout y de educación en el tiempo libre, estas experiencias no recibían el nombre de aprendizaje-servicio, sino que eran descritas como proyectos educativos con finalidad social o con servicio al entorno.

\section{Los primeros proyectos identificados como ApS}

En I'Hospitalet se empieza a hablar de aprendizaje-servicio a partir de 2004. En unos casos, se identifican y se bautiza como ApS experiencias que ya se llevaban a cabo sin este nombre. Podríamos decir que se "descubre" y se celebra su identidad ApS.

Un buen ejemplo es el proyecto Conocer es Amar, compartido desde el 2002 por el Instituto de Secundaria Bisbe Berenguer y la Escuela de Educación Especial Escorça. En una asignatura optativa dentro del área de Educación Física, los chicos y chicas de $3^{\circ}$ de educación secundaria obligatoria estimulan a chicos y chicas de educación especial, practicando con ellos juegos y ejercicios de deporte adaptado a fin de ayudarles a mejorar su motricidad. Éste ha sido y es un proyecto emblemático de la ciudad, inspirador de otras experiencias en todo el país.

En otros casos, los proyectos ya nacen bajo el paraguas conceptual del ApS. Un buen ejemplo fue la sistematización del proyecto JxB (Jóvenes por el Barrio), iniciado durante el curso 20072008 entre el Instituto de Secundaria Eduard Fontserè y el Esplai La Florida. En esta experiencia, ubicada en el Bachillerato (eduación secundaria no obligatoria), los jóvenes reciben una formación como voluntarios -reglada por la Generalitati- ubicada en la materia de Ética o de Prácticas de Empresa, y luego llevan a cabo un servicio como ayudantes de monitores en esplais o entidades sociales de la ciudad. Actualmente, esta experiencia se ha extendido a otros institutos y entidades y forma parte de la identidad pedagógica de la ciudad.

\section{Características del desarrollo del Aps en la ciudad}

La trayectoria de la generalización del aprendizaje-servicio en I'Hospitalet responde evolutivamente a un proceso ininterrumpido de ensayo -etapa piloto-, de estructuración y consolidación -etapa de crecimiento-y de saltar las fronteras -etapa de expansión-. Etapas, cada una de las cuales ha sumado los logros de la anterior.

\begin{tabular}{|l|l|}
\hline Etapa & Característica básica \\
\hline Etapa piloto & $\begin{array}{l}\text { Se analiza el impacto del } \\
\text { ApS en la educación para la } \\
\text { ciudadanía en un barrio con } \\
\text { dificultades sociales. Se } \\
\text { sientan las bases de una red } \\
\text { ciudadana para el impulso } \\
\text { del ApS en toda la ciudad. }\end{array}$ \\
\hline $\begin{array}{l}\text { Etapa de } \\
\text { crecimiento y } \\
\text { consolidación }\end{array}$ & $\begin{array}{l}\text { La organización de desarrolla } \\
\text { y estabiliza. Se generaliza e } \\
\text { intensifica la formación. Se }\end{array}$ \\
\hline
\end{tabular}




\begin{tabular}{|l|l|}
\hline & $\begin{array}{l}\text { ritualizan las actividades de } \\
\text { impulso al ApS en un plan de } \\
\text { actuación anual estable y se } \\
\text { intensifica su difusión. }\end{array}$ \\
\hline $\begin{array}{l}\text { Etapa de } \\
\text { expansión }\end{array}$ & $\begin{array}{l}\text { Se extiende el prestigio de la } \\
\text { ciudad como impulsora del } \\
\text { ApS en Cataluña, España y a } \\
\text { nivel internacional. }\end{array}$ \\
\hline
\end{tabular}

\section{Etapa piloto}

Esta primera etapa tiene lugar en dos cursos consecutivos: el 2007-2008 y el 2008-2009. Durante el primero de ellos el Departamento de Educación de la Generalitat de Cataluña impulsó el grupo de trabajo Educación para la Ciudadanía. Aprendizaje-Servicio. Centros y Entorno. Participaron más de 40 personas de centros escolares, entidades sociales y técnicos de la Administración Pública.

La finalidad de este grupo de trabajo era explorar el aprendizaje-servicio en la nueva asignatura de Educación para la Ciudadanía y en el marco de los llamados Planes Educativos de Entorno (políticas de apoyo educativo específico en barrios deprimidos). Docentes de l'Hospitalet, junto con educadores de entidades sociales y técnicos municipales, participaron de este grupo de trabajo, en el que también se añadieron otras ciudades que contaban con Planes Educativos de Entorno, como Granollers, Sabadell, Terrassa, y Sant Vicenç dels Horts, entre otros

Como consecuencia de esta operación, en el curso 2008-2009 l'Hospitalet fue una de las ciudades piloto escogida y analizada por el Departamento de Educación de la Generalitat (la otra fue Sant Vicenç dels Horts), para observar los resultados de la aplicación del aprendizaje-servicio en la entonces nueva asignatura de Educación para la Ciudadanía. Se trataba de analizar, pues, hasta qué punto era eficaz el aprendizaje-servicio en un barrio dotado con un Plan Educativo de Entorno y en el marco de la nueva asignatura. Se eligió el barrio de La Florida porque reunía dos ventajas. En primer lugar, la Florida contaba ya con centros educativos y entidades sociales con experiencias embrionarias de aprendizaje-servicio. En concreto, el Instituto de Secundaria Eduard Fontseré y el Esplai La Florida habían desarrollado ya una práctica ApS con chicos y chicas de Bachillerato (16-17 años) llamada Jóvenes por el Barrio y estaban empezando a programar otra práctica con chicos y chicas de Tercero de Secundaria (14-15 años) en el marco de la asignatura de Educación para la Ciudadanía, que era el objeto de la investigación de esta etapa piloto. En segundo lugar, el proyecto Jóvenes por el barrio, que en su momento había sido asesorado por el Centro Promotor de Aprendizaje Servicio de Catalunya, ya había empezado a destacar positivamente en alguna las líneas del Plan Educativo de Entorno del Barrio, concretamente en su potencial inclusivo respecto a los jóvenes más desfavorecidos. Eso llevaba a suponer que los buenos resultados se podrían generalizar.

De todos modos, se acordó desde el primer momento que, si bien la experiencia piloto se centraría en el barrio de La Florida, valía la pena abrirlo a otros barrios puesto que era capital que toda la ciudad se 
beneficiara de esta oportunidad.Para desarrollar esta experiencia piloto se articuló una red ciudadana, que en esta etapa piloto fue tutelada por la

Generalitat. Esta red tenía 4 nodos:

- Una coordinación institucional por parte de 3 personas: dos de ellas del Departamento de Educación de la Generalitat y la tercera pertenecía al Centro Promotor. Esta última ejercía también una función de coordinación técnica general.

- Un equipo de carácter técnico, Ilamado Grupo Permanente, para implementar y monitorizar las medidas y actuaciones que se iban decidiendo. En este equipo técnico participaron 7 personas: las tres anteriormente citadas, más 4 personas más entre técnicos de educación del ayuntamiento y de la formación de docentes en la ciudad.

- Un Grupo Territorial formado por 26 docentes y educadores sociales de la ciudad, que constituirían la base para llevar a cabo la formación y contrastar los pasos que se iban dando.

- Un plan de actuación, que se concretó en 5 bloques: formación, inventario, seguimiento, información y repercusión en medios. Las tareas o acciones realizadas en cada uno de ellos fueron un curso de introducción al aprendizaje-servicio; un inventario de experiencias ApS de la ciudad; el seguimiento y evaluación del proyecto ApS Compartimos Derechos impulsado conjuntamente por el Instituto de Secundaria Eduard Fontserè y el
Esplai La Florida en el marco de la asignatura de educación para la Ciudadanía; se informó al resto de centros de la ciudad mediante diversos recursos -como por ejemplo reuniones con directores y coordinadores pedagógicos de los centros educativos y la web del centro de formación del profesorado-. Asimismo el proyecto tuvo una repercusión significativa en los medios de comunicación a través del Diario de la ciudad, Televisión y Radio locales.

Dado que los resultados registrados por el Departamento de Educación de la Generalitat en esta etapa piloto fueron muy positivos, las personas de la ciudad que se habían implicado decidieron consolidar y ampliar de manera colaborativa la experiencia vivida, para construir entre todos una verdadera política pública de fomento del ApS, que fuera sostenible y que se basara en aprovechar lo que cada actor educativo y social podía aportar.

\section{Etapa de crecimiento y consolidación del ApS}

La segunda etapa en la generalización del ApS en I'Hospitalet se inicia en el curso 2009-2010 y finaliza en el 201314. Podemos decir que es a partir del 2009-2010 que el aprendizaje-servicio empezó a formar parte, de manera consistente, de la identidad pedagógica de la ciudad.

El Grupo Permanente, responsable de sacar adelante el plan de actuación anual, pasó a ser coordinado por una persona profesional del Área de Educación del ayuntamiento. El grupo 
ha oscilado entre 8 y 10 personas, se ha mantenido heterogéneo cuanto a procedencia de sus miembros

(Ayuntamiento, Centro de Formación del Profesorado, Centro Promotor de Aprendizaje Servicio, profesorado, entidades sociales, cooperativa de servicios ) y se ha regido siempre por el criterio de distribuir las tareas en función de lo que le es propio a cada miembro, procurando un funcionamiento muy ágil y operativo, orientado a resultados y repartiendo protagonismo y visibilidad.

Como elemento singular del Grupo Permanente en esta etapa hay que señalar los vínculos con otras redes, tanto locales como estatales, tal como expresa el gráfico siguiente:

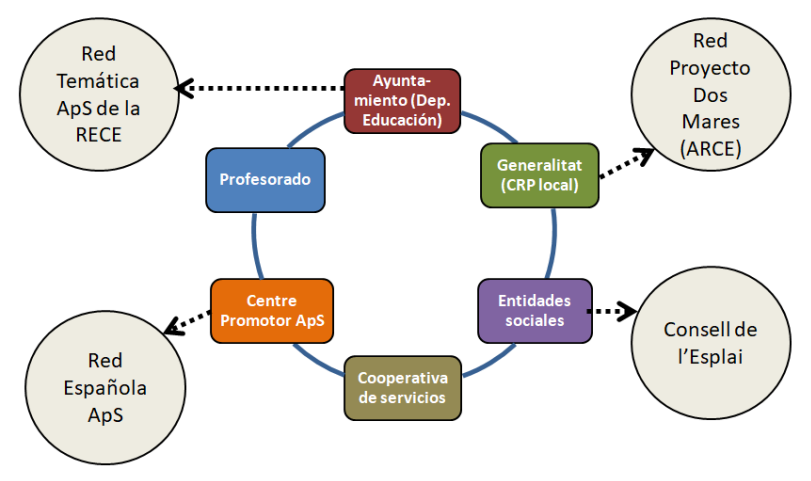

Estos vínculos actuaron a modo de ventanas abiertas a otras realidades, configurando de entrada un enfoque del aprendizaje-servicio nada endogámico, sino flexible y receptivo.

El plan de actuación ensayado en la etapa piloto no sólo se consolidó sino que se amplió, desplegándose paulatinamente más actividades.
Algunas de ellas se han convertido en tradicionales y otras han tenido un carácter más ocasional, pero de amplia repercusión. Destacamos a continuación las más significativas:

- En relación a la formación, se multiplicaron los cursos de iniciación al aprendizaje-servicio organizados desde el centro de recursos pedagógicos en los que participaron muchos docentes, en particular de la etapa de Secundaria y en menor medida, de Primaria y Formación Profesional. Además, la formación se abrió desde el principio a las entidades sociales, constituyendo un precedente insólito en nuestro país, donde lo más habitual era que en la formación de los docentes no tuvieran cabida otros perfiles.

- El Inventario de los proyectos ApS de la ciudad supone una tarea muy minuciosa, que no siempre avanza al mismo ritmo, pero que crece cada año. El inventario no sólo es un resultado tangible sino también una oportunidad de contactar con todos los actores educativos y sociales vinculados al aprendizaje-servicio.

- Después de la exitosa experiencia del asesoramiento a los proyectos Jóvenes por el Barrio y al proyecto Compartimos Derechos (asesorados ambos por el Centro Promotor de Aprendizaje Servicio), el siguiente fue el Proyecto Cicerone, una actuación de apadrinamiento entre estudiantes de Secundaria de la ciudad y chicos y chicas recién llegados, que fue asesorada por personal técnico del propio 
ayuntamiento.

- En otoño del 2010 tuvo lugar la primera Jornada de AprendizajeServicio de la ciudad, planteada como un punto de encuentro y de intercambio entre docentes, educadores de las entidades sociales y técnicos municipales. Desde entonces, cada año se ha ido repitiendo este evento, organizado desde el ayuntamiento. Como nota remarcable, desde el primer momento se invitó a ponentes internacionales aprovechando que estaban en Barcelona. Fue el caso de Alberto Croce (Argentina), Chantal Jouannet (Chile) o Mike Brough (California).

- Durante esta etapa los medios de comunicación de la ciudad han ido reflejando los diferentes proyectos, encuentros y jornadas.

Especialmente remarcable ha sido el compromiso de la televisión local, consiguiendo reforzar la relevancia de los proyectos de la ciudad. Cabe destacar la publicación de 15 vídeos por parte de las televisiones local y autonómica y del Centro Promotor de Aprendizaje Servicio de Catalunya

- Web-blog ApS de la ciudad. Es en esta etapa intermedia cuando empezamos a ensayar diferentes modelos de web para poder llegar mejor a los actores educativos y sociales de la ciudad. Estamos intentando encontrar el modelo sostenible cuanto al mantenimiento y actualización, y al mismo tiempo atractivo, pero hay que decir que todavía no lo hemos conseguido.

- La primavera del curso 2012-2013 se realiza la primera Fiesta de reconocimiento a los jóvenes de la ciudad que han estado participando en proyectos ApS. El acto tiene lugar por la tarde, en una discoteca de la ciudad, y cuenta con la actuación de un grupo musical merecedor de un premio local y con una entrega formal de diplomas que expresan este reconocimiento

- Es también durante estos años cuando empezamos a animarnos a publicar algunos documentos y a organizar presentaciones públicas que les dieran a conocer. A modo de ejemplo destacamos cuatro de los más significativos: el primero (octubre 2012) fue la sistematización del Proyecto JXB, Jóvenes por el barrio, fruto a su vez del asesoramiento realizado. La publicación fue posible gracias a un acuerdo entre el Consell de I'Esplai de l'Hospitalet (una federación de centros de tiempo libre de la ciudad), el Ayuntamiento de la ciudad y la Diputación de Barcelona. El segundo libro es Aprendizajeservicio en España. El contagio de una revolución pedagógica necesaria, de Roser Batlle, presentado en la ciudad el mes de febrero de 2014, actuando como invitada Ana Maria Scala, de la organización latinoamericana CLAYSS-sede Uruguay. Y más adelante (en marzo de 2015), el Centre d'Estudis de I'Hospitalet publicó Aprenentatge servei a I'Hospitalet de Llobregat. Ens fa 
crèixer. (Aprendizaje-servicio en I'Hospitalet de Llobregat. Nos hace crecer) una obra de Enric Roldán, compendio de historia, experiencias, testimonios, y aportaciones externas que ofrecen una visión muy completa del fenómeno del ApS en la ciudad. Por último, en 2016, el Centre d'Estudis de l'Hospitalet publicó la obra Participar a la ciutat:

El Consell de Nois i Noies de I'Hospitalet, (Participar en la ciudad: el Consejo de chicos y chicas de l'Hospitalet) cuyas autoras son Carolina Batet y Susagna Escardíbul.

- Durante los cursos 2011-2012 y 2012-2013 las visitas externas aumentan significativamente. Cuatro centros de formación del profesorado españoles (Santander, San Sebastián, Valencia y I'Hospitalet de Llobregat) compartieron un proyecto ARCE (Agrupaciones y Redes de Centros Educativos) impulsado por el Ministerio de Educación. Este proyecto común se articuló entorno al asesoramiento en aprendizajeservicio. La participación en este proyecto por parte del centro de recursos pedagógicos de l'Hospitalet representó una ventana abierta para difundir los avances de la ciudad, siendo ésta lugar de encuentro, visitas e intercambio en el marco del proyecto ARCE.

- En los cursos 2013-2014 y 20142015 se produjo un intercambio con educadores y adolescentes de la región del Trentino, en Italia. Tenían noticia del aprendizaje-servicio en la ciudad y deseaban explorar sus posibilidades. El primer año l'Hospitalet recibió la visita de 25 educadores italianos. Al poco tiempo nos visitó un colectivo de 160 jóvenes y 40 educadores italianos y al año siguiente fueron chicos y chicas de la ciudad a conocer las actividades de sus homólogos en Italia.

- También es en este período en el que, a partir de un acuerdo con la Universidad de Barcelona, se han llevado a cabo diversas investigaciones de impacto por parte de diversos grupos de investigación.

- Por último queremos destacar el apoyo político que surge en esta etapa de consolidación, en la que consiguen dos hitos importantes. Uno, la aprobación de una moción de apoyo al ApS por parte todos los grupos políticos en el pleno municipal el 27 de noviembre de 2012. Y dos, la incorporación de una cláusula en la convocatoria de subvenciones municipal que prioriza las acciones de ApS, desde el año 2012.

\section{Etapa de expansión}

Aunque la estrategia de impulso del ApS en la ciudad siempre ha sido abierta, flexible y expansiva, es a partir del curso 2014-2015 en adelante cuando alcanza un nivel mayor de visibilidad y reconocimiento a nivel catalán, español e internacional.

De hecho, los protagonistas de impulso del aprendizaje-servicio en I'Hospitalet 
nunca han esperado a consolidarse como grupo para abrirse a otras realidades. Ese mito, la necesitad de fortalecer lo interno como condición previa a abrirse a lo externo, está ciertamente arraigado en muchos sectores educativos y sociales, pero minusvalora el efecto de fortalecimiento interno que tiene el hecho de contactar con otros y abrirse al exterior. Es un efecto parecido a la movilización doméstica que provoca el tener que acoger invitados: se limpia y ordena la casa y con ello sale ganando la casa y no sólo los invitados.

\section{Durante esta etapa el Grupo}

Permanente ha quedado constituido por María José Pérez Triviño y Núria Ramos, del Área de Servicios a la Persona del Ayuntamiento; Manel Plens y Jordi Ibáñez, de los Servicios Educativos de la ciudad (Generalitat de Cataluña); Enric Roldán, profesor jubilado y miembro del Casalet (Movimiento de Renovación Pedagógica de la ciudad); Marina Lasaosa y Cristina Lope, de la Cooperativa Encís; Felipe Campos y Anna Cardona, del Consell de l'Esplai de l'Hospitalet y Roser Batlle, del Centro Promotor de Aprendizaje Servicio.

En este período la apuesta del ayuntamiento por impulsar una política pública de aprendizaje-servicio se ha fortalecido extraordinariamente y se ha erigido en España y a nivel internacional como un referente para las administraciones públicas. A título de ejemplo de compromiso municipal, actualmente el $40 \%$ de los proyectos ApS de la ciudad son apadrinados por otros servicios municipales distintos a la concejalía de Educación y, además, se cuenta con una dedicación parcial de 2 profesionales funcionarios y 2 personas contratadas.

En la etapa de expansión destacaríamos tres indicadores clave: la coordinación de la Red Temática Aprendizaje-Servicio como instrumento de cohesión social; el Premio Internacional Ciudad Educadora 2016; y la organización del $X$ Encuentro Estatal de Aprendizaje-Servicio.

1) En relación a la coordinación de la Red Temática Aprendizaje-Servicio como instrumento de cohesión social, está formada por 24 ayuntamientos españoles que persiguen fomentar el éxito educativo y el compromiso social de los niños, niñas y jóvenes de sus municipios, a través de implementar políticas públicas de fomento de las prácticas de aprendizaje-servicio. Liderada por el ayuntamiento de I'Hospitalet, es una red temática dentro de la Red Estatal de Ciudades Educadoras. Y a su vez, ésta se constituyó en 1996 como red territorial de la Asociación Internacional de Ciudades Educadoras. En el 2018 la RECE cuenta con 198 municipios adheridos.

La idea de crear una red temática de aprendizaje-servicio dentro de la RECE nace en el 2011, de la mano del ayuntamiento de Avilés. L'Hospitalet toma la coordinación en el 2014, pero es a partir del 2016 cuando adquiere consistencia. Bajo este liderazgo, reúne a un número significativo de ayuntamientos e inicia un trabajo colaborativo para fortalecer las políticas 
locales de ApS, trabajado juntos ayuntamientos veteranos en el impulso del ApS y ayuntamientos que quieren sumarse a él. En la red han coincidido ayuntamientos miembros de la RECE y ayuntamientos que todavía no son miembros.

La RECE se planteó como objetivos concretos realizar 3 encuentros anuales, coordinando agenda con los otros grupos de la RECE (noviembre 2016, abril 2017 y diciembre 2017) y construir colectivamente una "caja de herramientas": una colección de 8 guías prácticas para el impulso local del aprendizaje-servicio y compartirla con el resto de los ayuntamientos.

La elaboración de las guías prácticas se convirtió en el eje central del trabajo de la Red Aprendizaje-Servicio como instrumento de cohesión social. Para ello, el Ayuntamiento de l'Hospitalet buscó asesoramiento profesional en la Red Española de Aprendizaje-Servicio, que actuó a modo de coordinación técnica. Se elaboraron 8 guías entre finales del 2016 y principios del 2018 (Cómo crear un grupo local de impulso del ApS; Cómo promover formación ApS en el municipio; Cómo establecer alianzas con otros departamentos municipales; Cómo organizar una jornada local de ApS; Cómo reconocer a los niños, niñas y jóvenes protagonistas; Cómo incorporar el ApS en las convocatorias de subvenciones; Cómo elaborar un inventario de proyectos ApS locales; y, Cómo generar un espacio web de ApS).

Todos los encuentros se celebraron en I'Hospitalet de Llobregat, actuando como anfitrión el ayuntamiento de esta ciudad. Se organizaba cada encuentro en una mañana entera de trabajo y se finalizaba con un almuerzo para aquellas personas que podían o tenían que marchar un poco más tarde. Otro resultado tangible fue el acuerdo entre esta red de ayuntamientos y la Red Española de Aprendizaje-Servicio, por el cual cada año un ayuntamiento de la primera debería comprometerse a coorganizar el encuentro estatal que se celebra cada año, ininterrumpidamente desde el 2008.

2) El Premio Internacional Ciudad Educadora 2016. El 4 de junio de 2016 I'Hospitalet de Llobregat recogió uno de los tres premios Ciudades Educadoras, que por primera vez otorgaba la Asociación Internacional de Ciudades Educadoras. Mereció el premio compitiendo con otras 44 ciudades, de 11 países y 3 continentes. Ganaron tres ciudades: Espoo (Finlandia), Gamcheon de Saha-gu (República de Corea) y I'Hospitalet de Llobregat, en este caso por su política pública de impulso del aprendizaje-servicio. Este premio aportó mayor visibilidad y profundidad al reconocimiento internacional que l'Hospitalet, de hecho, había estado recogiendo poco a poco cada año, a través de las visitas de pedagogos españoles e italianos, ponencias de expertos internacionales, encuentros diversos y vínculos a otras redes.

3) La organización del X Encuentro Estatal de Aprendizaje-Servicio

En 2017 la ciudad coorganizadora y acogedora de esta cita anual del aprendizaje-servicio fue l'Hospitalet de 
Llobregat. El X Encuentro se ha celebró el viernes 1 de diciembre en el equipamiento municipal Centro de Actividades La Farga. El Encuentro, inaugurado por la alcaldesa Núria Marín, registró una asistencia de 458 personas, aunque inicialmente se habían previsto 300 . Esto representó un gran éxito cuanto a la afluencia de público. Cuanto a la procedencia, la distribución fue muy equilibrada entre participantes de la propia ciudad, del resto de Catalunya y del resto de España.

Durante este XI Encuentro tuvo lugar la entrega de Premios AprendizajeServicio 2017, que, en su tercera edición, incorporó también un premio específico al mejor proyecto de aprendizaje-servicio de la ciudad, que fue a parar al Proyecto Cicerone, presentado por 4 Institutos de Secundaria de la ciudad. Al final del evento, Ángel Viveros, alcalde de Coslada, recogió el testigo de Jaume Graells, concejal de educación de I'Hospitalet, para organizar el XI Encuentro Estatal de AprendizajeServicio del 2018 en Coslada (Madrid).

\section{Lecciones aprendidas}

A nivel de conclusiones y como factores determinantes que explican el desarrollo intenso del aprendizajeservicio en la ciudad podríamos citar:

- Vida asociativa intensa. Municipio ya de entrada particularmente activo en vida asociativa y redes educativas: terreno abonado para las alianzas que exigen los proyectos ApS y que garantizan su sostenibilidad.
- Voluntad política municipal:

Voluntad política clara por parte del ayuntamiento como primer responsable del impulso en el municipio. La labor del gobierno de la ciudad ha sido intensísima, durante esta década, en el apoyo y compromiso de impulso del aprendizaje-servicio. En este sentido, ha sido clave el compromiso de Lluis Esteve, actual Asesor Técnico de Educación y Teniente de Alcalde de Educación entre 2007y 2015 y de Jaume Graells, actual Teniente de Alcalde de Educación.

- Voluntad política supralocal: Contar con el apoyo político de otras Administraciones Públicas (Generalitat y Diputación de Barcelona) como elemento que ha favorecido la cohesión y ha otorgado legitimidad.

- Estructura de impulso heterogénea y participativa: Organización a través de una red local de impulso muy operativa (el Grupo Permanente y el Grupo Territorial) formada por personas de procedencia muy diversa; con elevado compromiso personal -no sólo con el aprendizaje-servicio, sino con la misma ciudad-; con tareas concretas y finalidades tangibles a conseguir; con buen entendimiento por parte de los profesionales (muy voluntarios) y los voluntarios (muy profesionales) que lideran estos grupos. El Grupo Permanente, en concreto, se ha esforzado siempre por compartir tareas, protagonismo y visibilidad. 
- Enfoque abierto, flexible y acogedor: El ApS se presentó desde el principio como reconocimiento a muchas prácticas que tanto los centros educativos como las entidades sociales ya llevaban a cabo. No se presentó como invento pedagógico sofisticado. Además, se percibió como estimulante el intercambio desde el primer momento con municipios y personas de otros países (españoles, italianos, argentinos, chilenos y uruguayos entre otros ), lo cual favoreció un posicionamiento modesto y al tiempo cosmopolita.

Además, hay que tener en cuenta un cierto efecto espiral: cada uno de estos factores refuerza y despliega los demás.

Como dice acertada y gráficamente Enric Roldán, fuimos capaces de poner el aprendizaje-servicio en el lado del corazón de la cabeza (Roldán, 2005).

\section{Referencias bibliográficas}

Graells, J., Esteve, L., Batlle, R. (2017). Experiencia primera: Sobre el aprendizaje-servicio como referencia de un trabajo sobre valores cívicos y desarrollo de la ciudadanía.

Ayuntamiento de I'Hospitalet. En J. Moya y F. Luengo (2017). Mejoras educativas en España (2017). Madrid: Anaya.

Roldán, E. (2005). Aprenentatge servei a l'Hospitalet de Llobregat. Ens fa crèixer. Hospitalet: Centre d'Estudis de l'Hospitalet.

\footnotetext{
i Se trata del Curso de Iniciación al Voluntariado,
}

Batlle, R. (2018). L'Hospitalet de Llobregat, modelo de generalización del aprendizaje-servicio. RIDAS, Revista Iberoamericana de Aprendizaje Servicio, 6, 54-68. DOI10.1344/RIDAS2018.6.7

habilitado en Catalunya para mayores de 16 años. 